\title{
Medicinal plants used in the treatment of maternal health-related problems by the Mapulana of Ehlanzeni District, Mpumalanga province, South Africa
}

\author{
Shalom Pabalelo Mashile*, Milingoni Peter Tshisikhawe, Ndivhaleni Anox Masevhe \\ Department of Botany, University of Venda, Thohoyandou 0950, South Africa.
}

\begin{tabular}{l}
\hline ARTICLE INFO \\
\hline Received on: $12 / 04 / 2019$ \\
Accepted on: 19/08/2019 \\
Available online: 03/12/2019 \\
\hline Key words: \\
Maternal healthcare, bulging, \\
sunken fontanelles.
\end{tabular}

sunken fontanelles.

\begin{abstract}
A decreased mortality rate among infants and children has been a health target for most health facilities. South Africa's free maternal healthcare services are not adequately utilized due to the tradition of new mothers following their parents' footsteps. The use of medicinal plant species in maternal healthcare has been perceived to be effective and reliable in preventing illness among communities. For this study, ethnobotanical data were collected by means of interviews using semi-structured questions responded to by the elderly, community adults, and youth who had been selected on the basis of referrals. Infants within the communities have been treated with traditional medicine for ailments, such as colic, nail biting, sunken, and bulging fontanelles. Hypoxis hemerocallidea Fisch., C. A. Mey. \& Ave-Lall, Lippia javanica (Burm.f.) Spreng., Vachellia karroo (Hayne) Banfi \& Glasso, and Annona senegalensis Pers. subsp. senegalensis were among plant species which were frequently reported as being used medicinally. The decoction was mostly prepared from the roots (79\%) followed by bulbs $(8 \%)$ and most of the decoction was taken orally. The study identified 13 plant species used successfully for the treatment of bulging and sunken fontanelles.
\end{abstract}

\section{INTRODUCTION}

Maternal healthcare has always been a topic in societies for decades, as they aim to reach international standards of decreased mortality rates of infants and children (Coovadia et al., 2009). South Africa is one of the countries that declared maternal healthcare free; including healthcare for lactating women and children under the age of six. The latter simply means that vaccinations and all requirements for pre-natal, infant birth, and post-natal stages are being funded by the government. These have been categorized as a priority in the healthcare system (Coovadia et al., 2009; Kibiribiri et al., 2016; Mhlanga, 2008). Dorrington et al. (2014) and statistical reports show a decrease in the number of infant mortality, from 40 to 30 per 1,000 infants and 56 to 40 per 1,000 children in 2011.

*Corresponding Author

Shalom Pabalelo Mashile, Department of Botany, University of Venda, Thohoyandou 0950, South Africa. E-mail: shalompabalelo@yahoo.com
An increased number of communities in South Africa still rely on traditional medicine for maternal health problems (Abdillahi and Van Staden, 2013; Randrianarivony et al., 2016). The reliance is due to tradition, whereby mothers follow in their parents' footsteps (Friend-du Preez et al., 2013). Shortages of medicine in public health facilities, cases where patients were referred to the pharmacy to purchase expensive medications, and wrong description of illness by mothers to health practitioners are among the reasons for the reliance. It is perceived that Traditional health practitioners not only rely on the mothers' diagnose but also on experience and spiritual diagnose (Friend-du Preez et al., 2013). The use of traditional medicines which are effective in preventing illnesses represents a significant area in the care-giving practices of mothers (Abdillahi and Van Staden, 2013; Bland et al., 2004). Paulos et al. (2016) further stipulated that traditional medicine is effective in preventing certain types of illness. Certain illness like inyoni, ibala are called African illnesses and are supposed to be remedied by traditional practices (Bland et al., 2004; Friend-du Preez et al., 2013). 
This study aimed at investigating medicinal plants used by the Mapulana in the treatment of certain maternal-health problems.

\section{MATERIALS AND METHODS}

\section{Study area}

The study was conducted in 15 villages in the Ehlanzeni District (Fig. 1) within three local municipalities, namely, Bushbuckridge, Mbombela, and Thaba Chweu. The Ehlanzeni district is among the three district municipalities located in the north-eastern part of Mpumalanga Province of South Africa.

\section{Data collection}

Structured questions were asked during interviews of participants selected for the study. The snowball technique was used whereby participants referred others for inclusion in the study (Cohen et al., 2007). The interviews were conducted face-to-face with selected elderly people, community adults, and youths. The interviews sought information on the names of various plants used to treat some common maternal-health problems, plant parts used, administration route, the traditional names for these ailments, and whether any ailments required combinations of plants. All participants signed prior consent forms as a show of willingness to participate in the study. Voucher specimens were collected for each plant species mentioned during the interviews were identified, labeled, and deposited in the University of Venda, Department of Botany's Herbarium. The researcher obtained ethical approval from the University of Venda Research Ethics Committee registered as project number SMNS/17/BOT/01/0905.

\section{Data analysis}

Thirty-eight participants were selected for the study and they were able to list various plants used for maternal-healthcare. A frequency index was calculated using a mathematical formula adapted from Madikizela et al. (2012). The formula $\mathrm{FI}=\mathrm{FC} / N \times 100$ states that $\mathrm{FI}$ is the frequency index, which expresses the percentage of frequency of listing a plant species by participants. FC is the number of participants who listed the use of a certain plant species and $N$ is the total number of participants.

\section{RESULTS AND DISCUSSION}

\section{Demography of informants}

Responses of participants are represented in Table 1, whereby community adults, were more knowledgeable about plants used for maternal care, followed by the elders. Fifteen percent of the overall participants were traditional health practitioner (THP). Overall, female participants responded well as compared to males, presumably because females are the recipient of maternal-healthcare. Most of the participants had at least attended secondary schools.

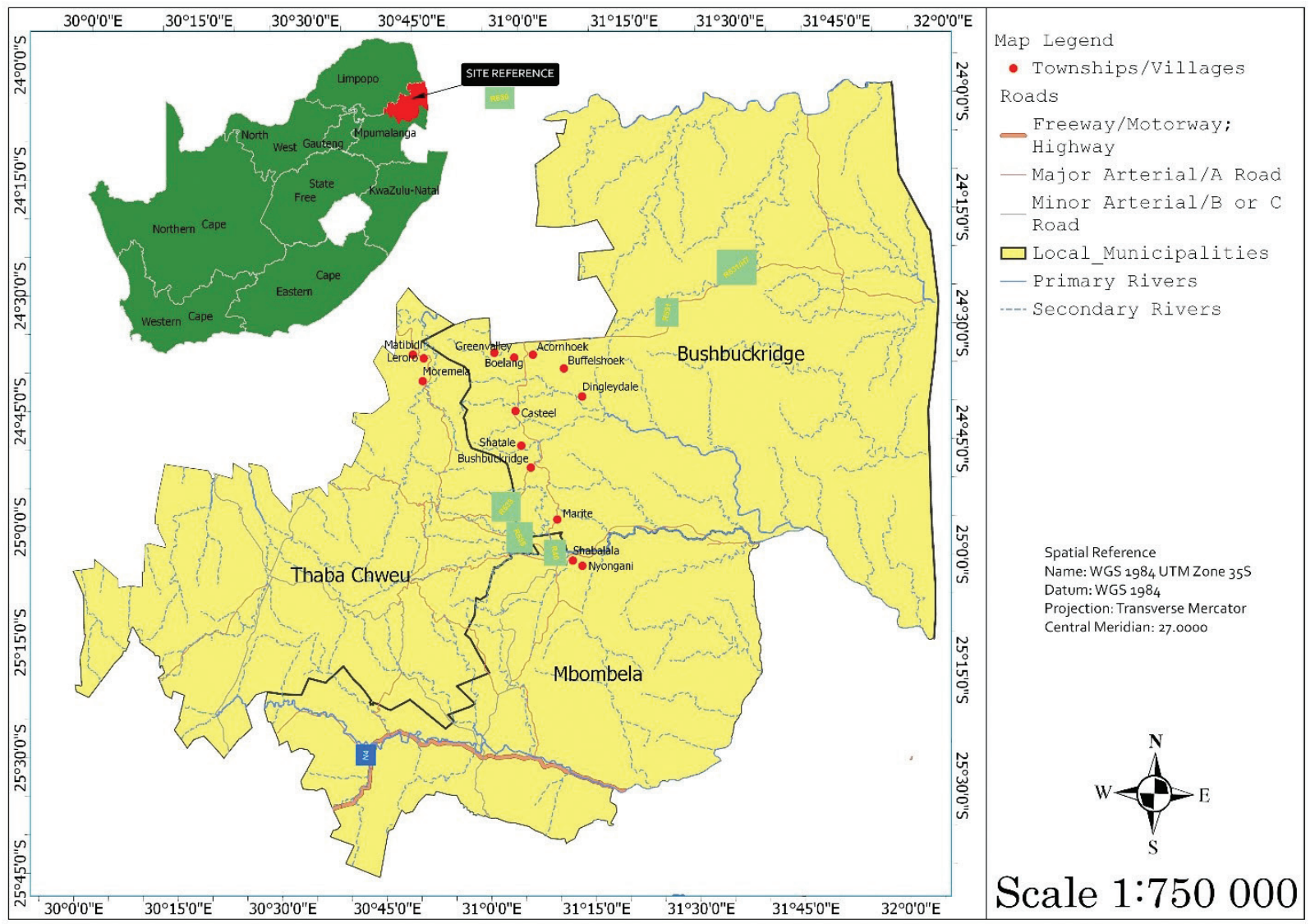

Figure 1. Showing map of Ehlanzeni local municipalities. 
Plant species used for the treatment of maternal healthcare problems

Thirty-two plant families with 49 genera used for maternal healthcare problems were recorded in the study (Table 2). Fabaceae was the most frequently cited family, followed by Apocynaceae, Asphodelaceae, and Rutaceae. Similar finding was observed by Steenkamp (2003); Mahwasane et al. (2013); Kankara et al. (2015); Yazbek et al. (2016). de Wet and Ngubane (2014) and Nduche et al. (2015) in their studies recorded Euphorbiaceae as the most cited families.

Plant parts frequently used in the current study were roots followed by bulbs, leaves, bark, and whole plant (Fig. 2). Similar finding was observed by Steenkamp (2003) and Mahwasane et al. (2013) where roots were the most used parts; however, Shosan et al. (2012); Nduche et al. (2015), and Kankara et al. (2015) reported leaves as the most frequently used parts, in their studies.

The frequently cited plant species were Annona senegalensis subsp. senegalensis and Senna occidentalis with

Table 1. Demographic structure of participants.

\begin{tabular}{llcc}
\hline Parameter & Specification & Frequency & Percentage (\%) \\
\hline \multirow{2}{*}{ Gender } & Male & 4 & 10.53 \\
& Female & 34 & 89.47 \\
Status & Youth (20-35) & 5 & 13.16 \\
& Community adult (36-59) & 18 & 47.37 \\
& Elder (60-99) & 15 & 39.47 \\
& None & 7 & 18.42 \\
& Primary & 10 & 26.32 \\
& Secondary & 17 & 44.74 \\
& Tertiary & 4 & 10.53 \\
\hline
\end{tabular}

$26 \%$ and $18 \%$ frequency index, respectively. Mahwasane et al. (2013) observed similar finding where A. senegalensis subsp. senegalensis had the highest frequency index in a study done among the Vhavenda of Lwamondo village in Limpopo Province of South Africa.

Decoctions were prepared and taken orally for almost all the plants mentioned in the study. Preparation of decoction was also highlighted by Abdillahi and Van Staden (2013) and Kankara et al. (2015). The mode of administration for all the plants as indicated in Table 3 depends on the instructions given by the THP or Herbalist and the nature or state of the problem (Mabogo, 1990; Steenkamp, 2003). For the treatment of conception problems, hematuria, and dysmenorrhea, mothers were usually required to drink half a cup, three times a day for 3 to 7 days of the decoction depending on the level of the problem. Babies were given oral medicine in small quantities, two to three times a day depending on their age. In the case of sunken or bulging fontanelles, the medication can be given in baby formula from day 1 of birth to 12 months of age or more if required or depending on the closure levels of the fontanelles. Typhoid treatment is rather unpleasant for children, since fresh leaves are crushed to make a paste which is inserted through the anus, however; oral mode is hence preferred.

Some plants are combined to increase the chances of healing; it is argued that all the plants species work synergistically.

Roots dominate in terms of being the plant parts utilized most for maternal healthcare purposes among the Mapulana. This is not a sound practice since it may affect the vigor of such plants thereby killing them. The death of individual plants ultimately affects the viability of the population in the long run and hence may lead to the demise of the species. Mapulana people avoid this by only harvesting the required roots from few trees or shrubs (Kurui et al., 2016).

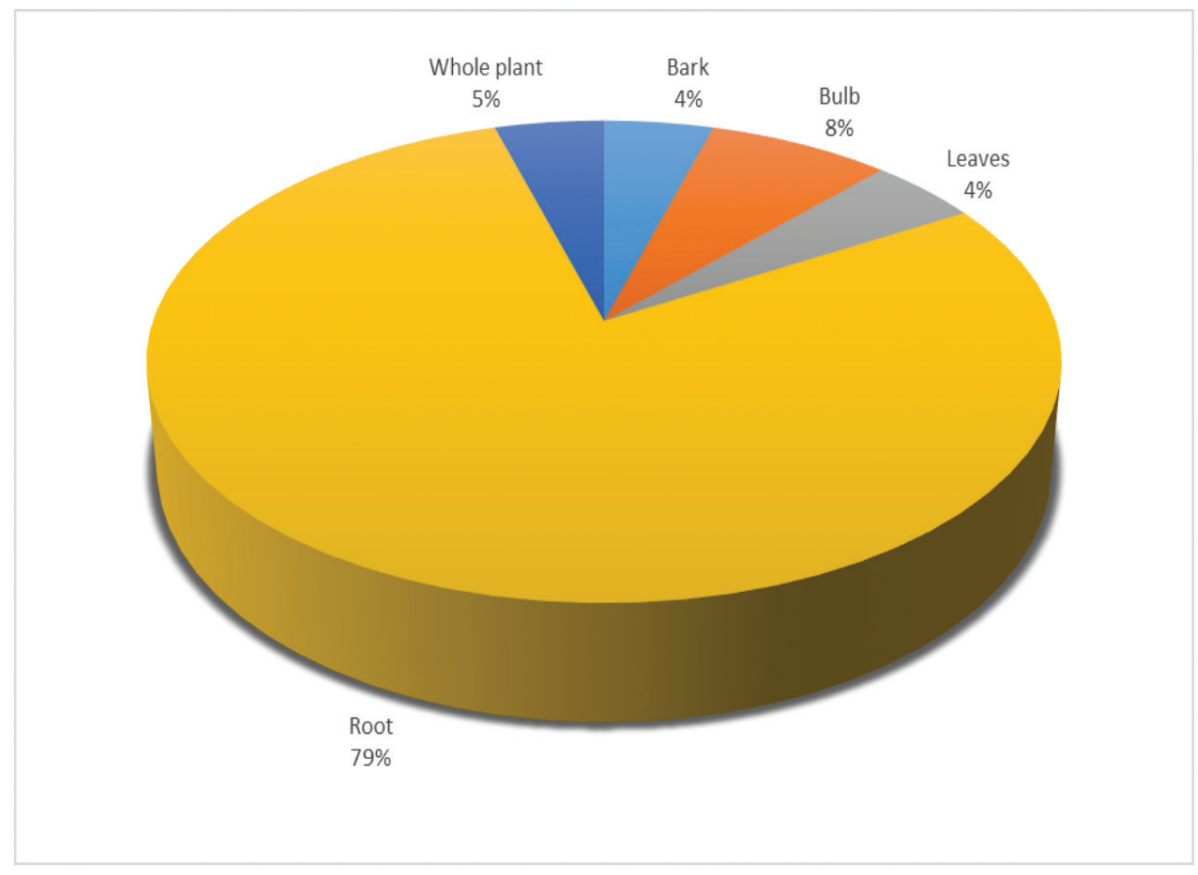

Figure 2. Plant part used for maternal healthcare. 
Table 2. Inventory of medicinal plants used in treatment of maternal health care in the Ehlanzeni District municipality.

\begin{tabular}{|c|c|c|c|c|c|c|}
\hline Family & Scientific name & Local name & Voucher number & Parts used & Mode of preparation & FI \\
\hline Cactaceae & Opuntia ficus-indica (L.) Mill. & Foyiyya & SP 084 & Roots & Decoction & 3 \\
\hline Passifloraceae & Adenia gummifera (Harv.) Harms var. gummifera & Komashatlha & SP 153 & Bulbs & Decoction & 3 \\
\hline Alliaceae & Allium sativum $\mathrm{L}$. & Konofolo & SP 092 & Leaves & Decoction & 3 \\
\hline Asphodelaceae & Aloe zebrina Baker & Legalane & SP 010 & Roots & Decoction & 3 \\
\hline Fabaceae & Senna occidentalis (L.) Link & Lenogane & SP 047 & Roots & Decoction & 18 \\
\hline Iridaceae & Cyperus latifolius Poir. & Lesegi & SP 046 & Roots & Decoction & 3 \\
\hline Agapanthaceae & Agapanthus africanus (L.) Hoffmanns. subsp. africanus & Letlladiane & SP 079 & Roots & Decoction & 11 \\
\hline Verbenaceae & Lantana rugosa Thunb. & Mabelemabutjwa & SP 082 & Roots & Decoction & 3 \\
\hline Polygaceace & Fallopia convolvulus (L.) Holub & Makgobata & SP 053 & Roots & Decoction & 3 \\
\hline Fabaceae & Erythrina lysistemon Hutch & Matlho ya baloi & SP 254 & Roots & Decoction & 5 \\
\hline Rubiaceae & Vangueria infausta Baker & Mmilo & SP 018 & Roots & Decoction & 3 \\
\hline Hypoxidaceae & Hypoxis hemerocallidea Fisch., C.A.Mey. \& Ave-Lall & Modimotsana wo mogolo & SP 067 & Roots & Decoction & 11 \\
\hline Hypoxidaceae & Hypoxis rigidula Baker var. rigidula & Modimotsane wo monyana & SP 077 & Roots & Decoction & 3 \\
\hline Fabaceae & Bauhinia galpinii N.E.Br & Mofethala & SP 042 & Roots & Decoction & 3 \\
\hline Vitaceae & Cyphostemma woodii (Gilg \& M.Brandt) Desc. & Mokgakgwa & SP 069 & Bark & Decoction & 3 \\
\hline Fabaceae & Piliostigma thonningii (Schumach.) Milne-Redh. & Mokgoropo & SP 176 & Roots & Decoction & 3 \\
\hline Fabaceae & Senna petersiana (Bolle) Lock & Mokorola kgogo & SP 174 & Roots & Maceration & 3 \\
\hline Lauraceae & Persea americana Mill. & Mokotapeni & SP 142 & Roots & Decoction & 3 \\
\hline Rutaceae & Citrus sinensis (L.) Osbeck & Monamona & SP 182 & Roots & Decoction & 3 \\
\hline Fabaceae & Cassia abbreviata Oliv. subsp. beareana (Holmes) Brenan & Monepenepo & SP 062 & Roots & Decoction & 3 \\
\hline Roseaceae & Prunus persica (L.) Batsch & Mopeta & SP 186 & Leaves & Decoction & 5 \\
\hline Caricaceae & Carica papaya $\mathrm{L}$. & Mophopho & SP 007 & Roots & Decoction & 5 \\
\hline Araliaceae & Cussonia transvaalensis Reyneke & Morotho & SP 171 & Roots & Bath & 3 \\
\hline Anacardiaceae & $\begin{array}{l}\text { Sclerocarya birrea (A.Rich.) Hochst. subsp. caffra (Sond.) } \\
\text { Kokwaro }\end{array}$ & Morula & SP 103 & Barks & Decoction & 11 \\
\hline Fabaceae & Vachellia karroo (Hayne) Banfi \& Glasso & Moseemane & SP 067 & Roots & Decoction & 3 \\
\hline Apocynaceae & Gomphocarpus fruticosus (L.) Aiton f. subsp. fruticosus & Moshekamolapo & SP 040 & Roots & Decoction & 3 \\
\hline Verbenaceae & Lippia javanica (Burm.f.) Spreng. & Moshukutjwane & SP 106 & Roots, leaves & Decoction & 3 \\
\hline Fabaceae & Abrus precatorius L. subsp. africanus Verdc. & Motatabaloyi & SP 041 & Roots & Decoction & 3 \\
\hline Fabaceae & Castanospermum australe A.Cunn. \& C.Fraser & Mothobesetsane & SP 059 & Roots & Decoction & 3 \\
\hline Apocynaceae & Carissa edulis (Forssk.) Vahl & Mothokolo & SP 183 & Roots & Decoction & 5 \\
\hline Olacaceae & Ximenia caffra Sond var. caffra & Motjhidi & SP 024 & Roots & Decoction & 8 \\
\hline Phyllanthaceae & Flueggea virosa (Roxb. ex Willd.) Voigt subsp. virosa & Motlhakawume & SP 045 & Roots & Decoction & 3 \\
\hline Ebenaceae & Euclea crispa (Thunb.) Gurke subsp. crispa & Motlhakola swifi & SP 055 & Roots & Decoction & 8 \\
\hline Ranunculaceae & Clematis brachiata Thunb. & Motlhemaphogo & SP 073 & Roots & smoke & 5 \\
\hline Annonaceae & Annona senegalensis Pers. subsp. senegalensis & Motllepo & SP 076 & Roots & Decoction & 26 \\
\hline Ebenaceae & Diospyros mespiliformis Hochst. ex A.DC. & Motsoma & SP 023 & Roots & Decoction & 3 \\
\hline Malvaceae & Grewia flavescens Juss & Mopharatshena & SP 043 & Roots & Decoction & 3 \\
\hline Vitaceae & $\begin{array}{l}\text { Cyphostemma cirrhosum (Thunb.) Desc. ex Wild \& } \\
\text { R.B.Drumm. subsp. cirrhosum }\end{array}$ & Sebabo & SP 113 & Roots & Decoction & 3 \\
\hline Orchidaceae & Ansellia africana Lindl. & Sefagama & SP 167 & Whole plant & Decoction & 5 \\
\hline Rutaceae & Zanthoxylum capense (Thunb.) Harv. & Semata & SP 050 & Roots & Decoction & 3 \\
\hline Celastraceae & Gymnosporia senegalensis (Lam.) Loes. & Sephashu & SP 052 & Roots & Decoction & 3 \\
\hline Zingiberaceae & Siphonochilus aethiopicus (Schweinf.) B.L.Burtt & Serokolo & SP 089 & Whole plant & Decoction & 11 \\
\hline Hyacinthaceae & Ledebouria revoulute (L.f.) Jessop & Sethuse & SP 097 & Roots & Decoction & 3 \\
\hline Solanaceae & Lycopersicon esculentum $\mathrm{L}$. & Tamatie & SP 267 & Leaves & Decoction & 5 \\
\hline Asphodelaceae & Bulbine frutescens (L.) Willd. & Tjhikwane & SP 268 & Bulbs & Decoction & 11 \\
\hline Lamiaceae & Leonotis ocymifolia (Burm.f.) Iwarsson & Unknown & SP 141 & Roots & Decoction & 3 \\
\hline Malvaceae & Sida acuta Burm.f. subsp. acuta & Unknown & SP 064 & Roots & Decoction & 3 \\
\hline Rubiaceae & Conostomium natalense (Hochst.) Bremek. var. natalense & Unknown & SP 063 & Roots & Decoction & 3 \\
\hline
\end{tabular}

FI denote the Frequency Index. 
Table 3. Administration of medicinal plants used in treatment of maternal health related symptoms in Ehlanzeni District municipality.

\begin{tabular}{|c|c|c|c|c|}
\hline Local treatment & Scientific name & Literature citations & Mode of administration & Recipient \\
\hline \multirow[t]{2}{*}{ Birth related issues } & Carissa edulis & $\begin{array}{l}\text { Stomach problems and venereal diseases } \\
\text { (Johns et al., 1990). }\end{array}$ & \multirow[t]{2}{*}{$\begin{array}{l}\text { Oral, half cup three times } \\
\text { a day }\end{array}$} & \multirow[t]{2}{*}{ Mother } \\
\hline & Opuntia ficus-indica & $\begin{array}{l}\text { Cleanse dirty blood (Semenya et al., 2013); sore breast } \\
\text { for nursing mothers (Deweck, 1997) and hair loss } \\
\text { (Bussmann and Glenn, 2010). }\end{array}$ & & \\
\hline Body weight & Cussonia transvaalensis & $\begin{array}{l}\text { Unknown, but C. spicata improves baby weight (Mabogo, } \\
\text { 1990). }\end{array}$ & Use for bath only three days & Baby \\
\hline \multirow[t]{3}{*}{ Colic } & Allium sativum & $\begin{array}{l}\text { Induce labor (Kamatenesi-Mugisha and Oryem-Origa, } \\
\text { 2007); restore fertility in men (Nduche et al., 2015) and } \\
\text { abdominal cramp (Shosan et al., 2012). }\end{array}$ & \multirow[t]{4}{*}{ Oral, give in small quantities } & \multirow[t]{4}{*}{ Baby } \\
\hline & Fallopia convolvulus & Unknown & & \\
\hline & Adenia gummifera var. gummifera & Unknown & & \\
\hline $\begin{array}{l}\text { Colic, sunken or bulging } \\
\text { fontanelles }\end{array}$ & Senna occidentalis & $\begin{array}{l}\text { Conception (Awai and Igoli, 2015); Stop bleeding during } \\
\text { pregnancy (Abdillahi and Van Staden, 2013). }\end{array}$ & & \\
\hline \multirow[t]{3}{*}{ Conception } & Castanospermum australe & Unknown & \multirow{4}{*}{$\begin{array}{l}\text { Oral, half cup three times } \\
\text { a day }\end{array}$} & \multirow[t]{4}{*}{ Mother } \\
\hline & Aloe zebrina & Herpes zoster (Chinsembu and Hedimbi, 2010) & & \\
\hline & Bauhinia galpinii & Food supplement (Mabogo, 1990). & & \\
\hline $\begin{array}{l}\text { Conception, stop bleeding } \\
\text { of pregnant women }\end{array}$ & Conostomium natalense var. natalense & Unknown & & \\
\hline \multirow[t]{2}{*}{ Dysmenorrhoea } & Cyperus latifolius & Unknown & \multirow{2}{*}{$\begin{array}{l}\text { Oral, half cup three times } \\
\text { a day }\end{array}$} & \multirow[t]{2}{*}{ Mother } \\
\hline & Piliostigma thonningii & $\begin{array}{l}\text { Stomach problems and haemotochezia (Mahwasane et al., } \\
\text { 2013). }\end{array}$ & & \\
\hline \multirow[t]{2}{*}{ Untie womb } & Grewia flavescens Juss & Infertility (Mabogo, 1990) & \multirow{2}{*}{$\begin{array}{l}\text { Oral, half cup three times } \\
\text { a day }\end{array}$} & \multirow[t]{2}{*}{ Mother } \\
\hline & Cassia abbreviata subsp. beareana & $\begin{array}{l}\text { Abortifacient (Steenkamp, 2003); C. fistula treats typhoid } \\
\text { (Shosan et al., 2012). }\end{array}$ & & \\
\hline \multirow[t]{2}{*}{ Hematuria } & Erythrina lysistemon & Labor pains (Grace et al., 2003). & \multirow{2}{*}{$\begin{array}{l}\text { Oral, half cup three times } \\
\text { a day }\end{array}$} & \multirow[t]{2}{*}{ Mother } \\
\hline & Hypoxis rigidula var. rigidula & Wounds and rash (Shale et al., 1999) & & \\
\hline Lerere & Vangueria infausta & $\begin{array}{l}\text { None, but } V \text {. apiculata induce labor (Kamatenesi-mugisha } \\
\text { and Oryem-origa, 2007) infertility (Mabogo, 1990; } \\
\text { Steenkamp, 2003). }\end{array}$ & Oral, give in small quantities & Baby \\
\hline \multirow[t]{2}{*}{ Nail-biting } & Acacia karroo & $\begin{array}{l}\text { General body health (Stafford et al., 2008), colic (Moteetee } \\
\text { and Van Wyk, 2011). }\end{array}$ & \multirow[t]{2}{*}{ Oral, give in small quantities } & \multirow[t]{2}{*}{ Baby } \\
\hline & Abrus precatorius subsp. africanus & Internal wound (Awai and Igoli, 2015). & & \\
\hline \multirow[t]{2}{*}{ Painful womb } & Ledebouria revoulute & Diarrhoea (Appidi et al., 2008) & \multirow{2}{*}{$\begin{array}{l}\text { Oral, half cup three times } \\
\text { a day }\end{array}$} & \multirow[t]{2}{*}{ Mother } \\
\hline & Cyphostemma woodii & Unknown & & \\
\hline $\begin{array}{l}\text { Prevent miscarriage } \\
\text { or still born, stopping } \\
\text { menstruation, Hiccups }\end{array}$ & Sclerocarya birrea subsp. caffra & $\begin{array}{l}\text { Infertility (Steenkamp, 2003); induce abortion (de Wet and } \\
\text { Ngubane, 2014); support pregnancy (Mabogo, 1990). }\end{array}$ & $\begin{array}{l}\text { Oral, bath and in hail smoke } \\
\text { depending on THP. Tie dry } \\
\text { fruit around a child's neck or }\end{array}$ & $\begin{array}{l}\text { Mother and } \\
\text { baby for hiccups }\end{array}$ \\
\hline \multirow{3}{*}{$\begin{array}{l}\text { Prevent miscarriage or } \\
\text { stillborn }\end{array}$} & Ximenia caffra var. caffra & Infertility (Steenkamp, 2003). & hands to stop hiccups & \\
\hline & Persea americana & $\begin{array}{l}\text { Used as contraceptive and sterilization for women } \\
\text { (Bussmann and Glenn, 2010) and fontanelles (Shosan et al., } \\
\text { 2012). }\end{array}$ & & \\
\hline & Citrus sinensis & Induce abortion (Nikolajsena et al., 2011) & & \\
\hline $\begin{array}{l}\text { Prevent miscarriage or } \\
\text { stillborn, typhoid }\end{array}$ & Siphonochilus aethiopicus & $\begin{array}{l}\text { Dysmenorrhea (Steenkamp, 2003); protect homestead } \\
\text { (Randrianarivony et al., 2016). }\end{array}$ & & \\
\hline Resuscitation, Hematuria & Carica papaya & Jaundice (Shosan et al., 2012; Tangjang et al., 2011). & \multirow{2}{*}{$\begin{array}{l}\text { Oral, bath and in hail smoke } \\
\text { depending on THP }\end{array}$} & \multirow[t]{2}{*}{ Baby } \\
\hline Resuscitation, nail-biting & $\begin{array}{l}\text { Agapanthus africanus subsp. } \\
\text { Africanus }\end{array}$ & Easy childbirth (Kaido et al., 1997). & & \\
\hline Sefetjwane & Senna petersiana & Infertility (Mabogo, 1990). & $\begin{array}{l}\text { Oral, give in small quantities } \\
\text { or incision on the chest }\end{array}$ & Baby \\
\hline $\begin{array}{l}\text { Stop bleeding of pregnant } \\
\text { women }\end{array}$ & Zanthoxylum capense & Infertility (Steenkamp, 2003). & $\begin{array}{l}\text { Oral, half cup three times } \\
\text { a day }\end{array}$ & Mother \\
\hline
\end{tabular}


Table 3. (Continued)

\begin{tabular}{|c|c|c|c|c|}
\hline Local treatment & Scientific name & Literature citations & Mode of administration & Recipient \\
\hline \multirow[t]{9}{*}{$\begin{array}{l}\text { Sunken or bulging } \\
\text { fontanelles }\end{array}$} & Hypoxis hemerocallidea & $\begin{array}{l}\text { Infants constipation (Masafu et al., 2016); infertility (Street } \\
\text { and Prinsloo 2013) and prevent premature abortion and } \\
\text { miscarriage (de Wet and Ngubane, 2014). }\end{array}$ & \multirow[t]{13}{*}{$\begin{array}{l}\text { Oral, give in small quantities } \\
\text { with other medication or } \\
\text { formula }\end{array}$} & \multirow[t]{13}{*}{ Baby } \\
\hline & Lantana rugosa & $\begin{array}{l}\text { Improves children's height (Mabogo, 1990; Mahwasane } \\
\text { et al., 2013) }\end{array}$ & & \\
\hline & Clematis brachiata & Headache (Mabogo, 1990) & & \\
\hline & $\begin{array}{l}\text { Gomphocarpus fruticosus subsp. } \\
\text { fruticosus }\end{array}$ & Headache and cold (Moteetee and Van Wyk 2011) & & \\
\hline & Diospyros mespiliformis & $\begin{array}{l}\text { Stomach problems (Mahwasane et al., 2013); D. lycyoides } \\
\text { infertility (Steenkamp, 2003); and D. spiliformis milk } \\
\text { enhancement (Kankara et al., 2015). }\end{array}$ & & \\
\hline & Ansellia Africana & Respiratory problems (Bandeira et al., 2001) & & \\
\hline & Sida acuta subsp. acuta & $\begin{array}{l}\text { Arrest threatened miscarriage (Nduche et al., 2015) } \\
\text { S. cordifolia induce labor (Randrianarivony et al., 2016) } \\
\text { and bleeding after delivery (Ogbe et al., 2009). }\end{array}$ & & \\
\hline & Leonotis ocymifolia & Stomach pains (w). & & \\
\hline & $\begin{array}{l}\text { Annona senegalensis subsp. } \\
\text { senegalensis }\end{array}$ & $\begin{array}{l}\text { Conception (Awai and Igoli, 2015); colic (Mabogo, 1990; } \\
\text { Mahwasane et al., 2013). }\end{array}$ & & \\
\hline \multirow[t]{3}{*}{$\begin{array}{l}\text { Sunken or bulging } \\
\text { fontanelles, conception }\end{array}$} & Euclea crispa var. crispa & $\begin{array}{l}\text { Dysmenorrhoea (Steenkamp, 2003) constipation in children } \\
\text { (Chinsamy and Koitsiwe, 2016). }\end{array}$ & & \\
\hline & Gymnosporia senegalensis & Infertility (de Wet and Ngubane, 2014). & & \\
\hline & Lippia javanica & Diarrhoea (van Vuuren et al., 2015) & & \\
\hline $\begin{array}{l}\text { Sunken or bulging } \\
\text { fontanelles, typhoid }\end{array}$ & Bulbine frutescens & Unknown & & \\
\hline \multirow[t]{2}{*}{ Typhoid } & Prunus persica var. persica & Antidiarrhoeal usage (Madikizela et al., 2012) & \multirow{2}{*}{$\begin{array}{l}\text { Oral, give in small quantities } \\
\text { or Insert as paste through } \\
\text { the anus }\end{array}$} & \multirow[t]{2}{*}{ Baby } \\
\hline & Lycopersicon esculentum & Induce labor (Kamatenesi-Mugisha and Oryem-Origa, 2007) & & \\
\hline
\end{tabular}

\section{Local conditions treated by Mapulana}

Phogwana

The term Phogwana represents sunken or bulging fontanelles which are usually called "soft spots" on the infants' head; the treatment is over the first 12 months of the baby's life. The process is called "cleansing period" (Bland et al., 2004). Fontanelles or ukhakhanyi, inyoni (as called by Zulu-speaking people), are perceived as an open entrance for evil spirits, hence warranting immediate treatment (Cocks and Moller, 2002). Mapulana infants are treated with traditional medicine for sunken and bulging fontanelles. The study identified 13 plant species that treat the ailment-Hypoxis hemerocallidea, S. occidentalis, Annona senegalensis subsp. senegalensis, Lantana rugosa, Clematis brachiate, Gomphocarpus fruticosus subsp. fruticosus, Diospyros mespiliformis, Ansellia Africana, Leonotis ocymifolia, Euclea crispa var. crispa, Flueggea virosa, Gymnosporia senegalensis, Sida acuta, and Lippia javanica. Within these plants species, there are combinations used for the treatment as indicated in Table 4. Our findings supported that of Mabogo (1990) who identified the use of A. senegalensis subsp. senegalensis in the treatment of fontanelles among the Vhavenda people. Persia americana was one of the species recorded by Shosan et al. (2012) to treat fontanelles in Nigeria, while Abdillahi and Van Staden (2013) recorded Ormocarpum trichocarpum as a treatment for fontanelles in some parts of South Africa.

The sunken and bulging fontanelles cleansing purposes in infants include when they have diarrhea. Diospyros mespiliformis was reported as treating diarrhea in Lwamondo (Mahwasane et al., 2013); similar finding includes using G. senegalensis for treating diarrhea by de Wet and Ngubane (2014) and Vuuren et al. (2015) which also quantifies the cleansing period, since some of the
Table 4. Combination of medicinal plants species used for maternal health care.

\begin{tabular}{lll}
\hline Plants species & Part used & Treatment \\
\hline Lippia javanica + Prunus persica & Leaves & Typhoid \\
$\begin{array}{l}\text { Prunus persica }+ \text { Lycopersicon esculentum } \\
\text { Diospyros mespiliformis }+ \text { Euclea crispa var } \\
\text { crispa }\end{array}$ & Roots & $\begin{array}{l}\text { Sunken and bulging } \\
\text { fontanelles }\end{array}$ \\
$\begin{array}{l}\text { Senna occidentalis }+ \text { Annona senegalensis } \\
\text { Euclea crispa }+ \text { Carica papaya }\end{array}$ & & \\
\hline
\end{tabular}

species used for sunken and bulging fontanelles are also used to minimize diarrhea.

\section{Lenogane}

The term Lenogane refers to the weak intestine as a result of an internal wound not healing after the baby's umbilical cord was removed. Treatment of abdominal pains (colic) in infants including stomach sounds is referred to as inkaba in Zulu (Bland et al., 2004). Allium sativum, S. occidentalis, and Fallopia convolvulus are the only species recorded for treating colic. Healing of wound is also supported by food supplement prepared with Bauhinia galpinii (Mabogo, 1990). The food supplement can in turn also facilitate relief from constipation. In Botswana, E. crispa var. crispa was reported to relieve constipation (Chinsamy and Koitsiwe, 2016). Masafu et al. (2016) in their comparative studies of medicinal plants used in Thulamela and JS Moroka Municipalities of South Africa reported that H. hemerocallidea was used in the treatment of constipation and intestinal parasites. They also reported the use of Mewilla plumbea in the treatment of colic. Tephrosia purpurea subsp. purpurea is a treatment for 
internal wounds for women in Benue State, Nigeria (Awai and Igoli, 2015). Moteetee and Van Wyk (2011) recorded Equisetum ramosissimum and Acacia karroo as the treatment for colic in Lesotho. Masafu et al. (2016) recorded M. plumbea being used as the treatment for colic from a study of the natural habitats and the uses of medicinal plants in Thulamela and JS Moroka Municipalities. Allium sativum was recorded in Abeokuta South Local Government Area of Ogun State, Nigeria, as one of the species treating abdominal cramps (Shosan et al., 2012).

\section{Motlhapo}

Mohlapo is a term used when women have blood in their urine (hematuria). The plant species identified from the study were Erythrina lysistemon and Hypoxis rigidula var. rigidula.

Metjhwa

When babies frequently want to feed or are constantly licking their nails, the ailment is called Metjha (nail-biting). Acacia karroo and Abrus precatorius subsp. africanus are the two species identified as being used for the treatment of the condition by the Mapulana.

\section{Ditshemane or Manogane or Nkusu}

Ditshemane or Manogane or Nkusu is the local name referring to typhoid among the Mapulana. The treatment is applicable to children of all ages. Leaves of Prunus persica var. persica and Lycopersicon esculentum are used as in combination to treat typhoid. Other combinations are shown in Table 4. Shosan et al. (2012) recorded four species for treating typhoid, including Mangifera indica and Sorghum bicolor.

Selomi

Selomi is when women experience severe period pains (dysmenorrhea) and some of the women are unable to conceive as a result. It is perceived that most women with Selomi have "tied-up wombs." The participants also argued that the tying of the womb can also be spiritually connected. Dysmenorrhea is treated with Cyperus latifolius and Piliostigma thonningii. In a study conducted in Lwamondo by Mahwasane et al. (2013), P. thonningii and D. mespiliformis were reported as being used in the treatment of stomach problems. The treatment of dysmenorrhea traditionally was recorded from numerous studies. Steenkamp (2003), Mahwasane et al. (2013), and de Wet and Ngubane (2014) have recorded many plant species used for the treatment of dysmenorrhea. Senna occidentalis and E. crispa var. crispa were also identified in the treatment of dysmenorrhea by Mabogo (1990), Steenkamp (2003) and Moteetee and Van Wyk (2011).

Aloe zebrine, Castanospermum australe, B. galpinii, and Conostomium natalense var. natalense are recorded species for the treatment of conception difficulties. Awai and Igoli (2015) recorded 10 plant species for the treatment of conception in Benue State, Nigeria, including A. senegalensis subsp. senegalensis, Carica papaya, and S. occidentalis. Masafu et al. (2016) reported that $H$. hemerocallidea was used in the treatment of infertility. From the current study, Grewia flavescens and Cassia abbreviata plant species were reported to "untie women's wombs". Rhamnus mucronata, Casearia gladiiformis, and Scolopia stolzii were also reported for treatment of conception difficulties in Dabaga Ulongambi Forest Reserve, Tanzania (Kitula, 2007).

\section{Makgoma}

Makgoma is a term used to describe the results of people's negligence of their tradition. The consequences include swollen stomach or body parts (legs and hands), coughing, and a shiny appearance. In this study, the researcher's concentration with the issue was in relation to infants and pregnant women. In the case of pregnant women, they can be affected by Makgomo if they do not follow all the rules in their tradition. Certain rules, such as pregnant women being restricted from having sexual relations when there is a funeral in their families; they are expected to wait for 7 days of cleansing before any intimacy. In addition, if a woman has an illicit relationship and then cooks for people at her family funeral, whoever eats the food will become "contaminated" "o kgomisiwe". Sclerocarya birrea subsp. caffra, Ximenia caffra var. caffra, Persea americana, Siphonochilus aethiopicus, and Citrus sinensis were the recorded species used to treat Makgoma in women. Nduche et al. (2015) recorded S. acuta subsp. acuta as arresting a threatened miscarriage. Bauhinia petersiana was recorded as preventing miscarriage in Mulanje District, Malawi (Maliwichi-Nyirenda and Maliwichi, 2010). Abdillahi and Van Staden (2013) have recorded Sasevieria hycacinthoides and Pyrenacantha scandens in the prevention of miscarriage in South Africa. Anyi-Ndenye women of Eastern Cote d'Ivoire have several plant species used to prevent miscarriages within the three trimesters of pregnancy. Some of the species recorded for this use were Desmodium adscendens, Ficus exasperate, and Cyanthyla prostrate (Malan and Neuba, 2011).

Sclerocarya birrea subsp. caffra is used to support pregnancy (Mabogo, 1990) and prenatal care (Kankara et al., 2015; Randrianarivony et al., 2016). Siphonochilus aethiopicus is used to protect homesteads (Masafu et al., 2016). People living around the Analavelona forest in South West Madagascar use a combination of three to ten plant species for protecting women and babies from evil spirits. Plant species used are Sida cordifolia, Flacourtia ramontchi, and Grewia flavascens to mention a few (Randrianarivony et al., 2016). Similar results were observed by Cocks and Moller (2002) where women bought plant medicines for the protection of their infants.

\section{Difeka}

Difeka is connected with Makgoma, whereby women's negligence might cause the baby to be instantly disabled or disabled after 20 months of birth and turn into an infant. This can be caused by the negligence of tradition by ignoring the generic protocol. For instance, if a third-born woman has intercourse on a firstborn pregnant woman's bed, when the pregnant woman later uses the bed "o fikegele" might occur, that is the pregnant woman may deliver a disabled child. In the case of a woman who had lost a baby and commits "di fikeka" this might result in that woman not conceiving or death. Agapanthus africanus and C. papaya were reported as remedying difeka. Agapanthus africanus subsp. africanus is taken for prolonged labor (Abdillahi and Van Staden, 2013; Kaido et al., 1997; Steenkamp, 2003), and C. papaya induce lactation (Randrianarivony et al., 2016). 


\section{CONCLUSION}

Traditional medicine is still relied upon in the treatment of some of the ailment in the study, especially for sunken and bulging fontanelles. A large number of medicinal plants reported to be used in the treatment of maternal healthcare warrant further research to validating such reliance and claims. Overuse of roots as medicinal materials is also a cause for concern in terms of the sustainability of natural resource utilization.

\section{ACKNOWLEDGMENT}

The University of Venda and National Research Foundation are acknowledged for providing funding. Participants are thanked for sharing their valuable information. Dr. PK Kaburise is appreciated for editing the document.

\section{CONFLICT OF INTEREST}

Mashile SP, Tshisikhawe MP, and Masevhe NA have no interest of conflict.

\section{FUNDING SOURCES}

The University of Venda (Project number SMNS/17/ BOT/01) and National Research Foundation (Grant number 102082) have funded the study.

\section{REFERENCES}

Abdillahi HS, Van Staden J, Application of medicinal plants in maternal healthcare and infertility: A South African perspective. Planta Med, 2013; 79:591-9.

Appidi JR, Grierson, DS, Afolayan AJ. Ethnobotanical study of plants used for the treatment of diarrhoea in the Eastern Cape. Pak J Biol Sci, 2008; 15:1961-3.

Awai EP, Igoli JO, Medicinal plants used in antenatal and perinatal care among the tiv people of Benue State, Nigeria. Indo Glob J Pharm Sci, 2015; 5:90-3.

Bandeira S, Gaspar F, Pagula FP. Ethnobotany and healthcare in Mozambique. Pharm Biol 2001; 39(1):70-3.

Bland R, Rollins N, Van Den Broeck J, Coovadia H, The use of non-prescribed medication in the first 3 months of life in rural South Africa. Trop Med Int Heal, 2004; 9:118-24.

Bussmann RW, Glenn A. Medicinal plants used in Northern Peru for reproductive problems and female health. J Ethnobiol Ethnomed, 2010; 6:30.

Chinsamy M, Koitsiwe M. Traditional knowledge of medicinal and food plant uses for sustainable community livelihoods: a case of Batswana communities in South Africa. J Soc Sci, 2016; 46:146-53.

Chinsembu K, Hedimbi M. An ethnobotanical survey of plants used to manage HIV/AIDS opportunistic infections in Katima Mulilo, Caprivi region, Namibia. J Ethnobiol Ethnomed, 2010; 6:25.

Cocks M, Moller V. Use of indigenous and indigenised medicines to enhance personal well-being: a South African case study. Soc Sci Med, 2002; 54:387-97.

Cohen L, Manion L, Morrison K. Research methods in education. 6th edition, Taylor and Francis or Routledge, London and New York, 2007.

Coovadia H, Jewkes R, Barron P, Sanders D, McIntyre D. The health and health system of South Africa: historical roots of current public health challenges. Lancet, 2009; 374:817-34.

de Wet, H, Ngubane SC. Traditional herbal remedies used by women in a rural community in northern Maputaland (South Africa) for the treatment of gynaecology and obstetric complaints. South African J Bot, 2014; 94:129-39.

Deweck AC. Ethnobotanical use of plants, part 4: The American continent. Cosmet Toiletries, 1997; 112:4.
Dorrington R, Bradshaw D, Laubscher R. Rapid mortality surveillance report, pp 1-17, 2014. ISBN: 978-1-920618-00-1

Friend-du Preez N, Cameron N, Griffiths P. "So they believe that if the baby is sick you must give drugs." The importance of medicines in health-seeking behaviour for childhood illnesses in urban South Africa. Soc Sci Med, 2013; 92:43-52.

Grace OM, Prendergast HDV, Jäger AK, Van Staden J. Bark medicines used in traditional healthcare in KwaZulu-Natal, South Africa: an inventory. South Afr J Bot, 2003; 69:301-3.

Johns T, Kokwaro JO, Ebi KK, Herbal remedies of the Luo of Siaya District, Kenya: establishing quantitative criteria for consensus. Econ Bot, 1990; 44:369-81.

Kaido TL, Veale DJ, Havlik I, Rama DBK. Preliminary screening of plants used in South Africa as traditional herbal remedies during pregnancy and labour. J Ethnopharmacol, 1997; 55:185-91.

Kamatenesi-Mugisha M, Oryem-Origa H. Medicinal plants used in some gynaecological morbidity ailments in western Uganda. Afr J Ecol, 2007; 45:34-40.

Kamatenesi-Mugisha M, Oryem-Origa H. Medicinal plants used to induce labour during childbirth in western Uganda. J Ethnopharmacol, 2007b; 109:1-9.

Kankara S, Ibrahim M, Mustafa M, Go R. Ethnobotanical survey of medicinal plants used for traditional maternal healthcare in Katsina State, Nigeria. South African J Bot, 2015; 97:165-75.

Kibiribiri E, Moodley D, Groves AK, Sebitloane, MH. Exploring disparities in prenatal care between refugees and local South African women. Int J Gynecol Obstet, 2016; 132:151-5.

Kitula RA. Use of medicinal plants for human health in Udzungwa Mountains Forests: a case study of New Dabaga Ulongambi Forest Reserve, Tanzania. J Ethnobiol Ethnomed, 2007; 3:2-5.

Kurui CM, Kiptui M, Chelang'a JK, Omondi P. Traditional controls of harvesting and conserving medicinal plants in Keiyo South SubCountry, Kenya. Int J Humanit Soc Sci, 2016; 6:54-63.

Mabogo EDN. The ethnobotany of the Vhavenda. MSc Dissertation University Pretoria, Pretoria, 1990

Madikizela B, Ndhlala A, Finnie JF, Van Staden J. Ethnopharmacological study of plants from Pondoland used against diarrhoea. J Ethnopharmacol, 2012; 141:61-71.

Mahwasane ST, Middleton L, Boaduo N, An ethnobotanical survey of indigenous knowledge on medicinal plants used by the traditional healers of the Lwamondo area, Limpopo province, South Africa. South African J Bot, 2013; 88:69-75.

Malan DF, Neuba DFR. Traditional practices and medicinal plants use during pregnancy by Anyi-Ndenye women (Eastern Cote d'Ivoire). Afr J Reprod Health, 2011; 15:85-93.

Maliwichi-Nyirenda CP, Maliwichi LL. Medicinal plants used for contraception and pregnancy-related cases in Malawi: a case study of Mulanje District. J Med Plants Res, 2010; 4:2121-7.

Masafu MM, Mbajiorgu CA, Nemadodzi LE, Kabine ES. A study of natural habitats and uses of medicinal plants in Thulamela and JS Moroka Municipalities, South Africa. Indian J Tradit Knowl, 2016; 15:363-9.

Mhlanga RE. Maternal, newborn and child health: 30 years on. South Afr Heal Rev, 2008; 2008:115-28.

Moteetee A, Van Wyk, BE. The medical ethnobotany of Lesotho: a review. Bothalia, 2011; 41:209-28.

Nduche MU, Omosun G, Okwulehie IC, Ethnobotanical survey of plants used as remedy for fertility conditions in Ebonyi State of Nigeria Sch Acad J Biosiences, 2015; 3:214-21.

Nikolajsena T, Nielsena F, Rasch V, Sørensenc PH, Ismaild F, Kristiansene U, Jägera A. Uterine contraction induced by Tanzanian plants used to induce abortion. J Ethnopharmacol, 2011; 137:921-5.

Ogbe FMD, Eruogun OL, Uwagboe M. Plants used for female reproductive health care in Oredo local government area, Nigeria. Sci Res Essays, 2009; 4:120-30.

Paulos B, Fenta TG, Bisrat D, Asres K, Health seeking behavior and use of medicinal plants among the Hamer ethnic group, South Omo 
zone, southwestern Ethiopia. J Ethnobiol Ethnomed, 2016; 12:44; doi:10.1186/s13002-016-0107-x

Randrianarivony $\mathrm{T}$, Randrianasolo A, Andriamihajarivo T, Ramarosandratana AV, Jeannoda VH, Rakotoarivony F, Bussmann RW, Useful plants and tradition for pregnancy, child delivery and for post-partum care used by people living around Analavelona Forest in South West Madagascar. Indian J Tradit Knowl, 2016; 15:68-78.

Semenya SS, Potgieter MJ, Erasmus LJC, Exotic and indigenous problem plants species used, by the Bapedi, to treat sexually transmitted infections in Limpopo Province, South Africa. Afr Health Sci, 2013; 13:320-6.

Shosan LO, Fawibe OO, Ajiboye AA, Abeegunrin T, Agboola DA. Ethnobotanical survey of medicinal plants used in curing some diseases in infants in Abeokuta South Local Government Area of Ogun State, Nigeria. Am J Plant Sci, 2012; 5:3258-68.

Stafford GI, Pedersen ME, Van Staden J, Jäger AK. Review on plants with CNS-effects used in traditional South African medicine against mental diseases. J Ethnopharmacol, 2008; 119:513-37.

Steenkamp V. Traditional herbal remedies used by South African women for gynaecological complaints. J Ethnopharmacol, 2003; 86:97-108.
Street RA, Prinsloo G. Commercially important medicinal plants of South Africa: a review. J Chem, 2013:1-16.

Tangjang S, Namsa N, Aran C, Litin A, An ethnobotanical survey of medicinal plants in the Eastern Himalayan zone of Arunachal Pradesh. India J Ethnopharmacol, 2011; 34:18-25.

van Vuuren SF, Nkwanyana MN, de Wet H. Antimicrobial evaluation of plants used for the treatment of diarrhoea in a rural community in South Africa. Complement Altern Med, 2015; 15:1-8.

Yazbek PB, Tezoto J, Cassas F, Rodrigues E, Plants used during maternity, menstrual cycle and other women's health conditions among Brazilian cultures. J. Ethnopharmacol, 2016; 179:310-31.

\section{How to cite this article:}

Mashile SP, Tshisikhawe MP, Masevhe NA. Medicinal plants used in the treatment of maternal health-related problems by the Mapulana of Ehlanzeni District, Mpumalanga province, South Africa. J Appl Pharm Sci, 2019; 9(12):021-029. 